\title{
Cancer cachexia as a determinant of efficacy of first-line pembrolizumab in patients with advanced non-small cell lung cancer
}

\author{
HIRONORI FUJII $^{1}$, AYUMU ARAKI ${ }^{1}$, HIROTOSHI IIHARA ${ }^{1}$, DAIZO KAITO ${ }^{2}$, CHIEMI HIROSE $^{1}$, \\ MOTOHIKO KINOMURA ${ }^{1}$, MIZUKI YAMAZAKI ${ }^{1}$, JUNKI ENDO ${ }^{2}$, TOSHIYA INUI ${ }^{2}$, KOMEI YANASE ${ }^{2}$, \\ YUKA SASAKI $^{2}$, TAKENOBU GOMYO ${ }^{2}$, CHIZURU SAKAI ${ }^{2}$, DAISUKE KAWAE ${ }^{2}$, YU KITAMURA ${ }^{2}$, \\ MASACHIKA FUKUI $^{2}$, RYO KOBAYASHI ${ }^{1}$, YASUSHI OHNO ${ }^{2}$ and AKIO SUZUKI ${ }^{1}$ \\ ${ }^{1}$ Department of Pharmacy, Gifu University Hospital; ${ }^{2}$ Department of Cardiology and Respirology Medicine, \\ Gifu University Graduate School of Medicine, Gifu 501-1194, Japan
}

Received November 19, 2021; Accepted January 12, 2022

DOI: $10.3892 / \mathrm{mco} .2022 .2524$

\begin{abstract}
Pembrolizumab, either as a type of monotherapy or in combination with cytotoxic anticancer agents, is effective in the treatment of advanced non-small cell lung cancer (NSCLC). However, the development of cancer cachexia may adversely affect anticancer drug therapy. The present study investigated the effect of cancer cachexia on clinical outcomes in patients with advanced NSCLC who received first-line pembrolizumab. The data of patients with advanced NSCLC receiving first-line monotherapy or combination therapy with pembrolizumab were retrospectively analyzed. The primary endpoint was time to treatment failure (TTF), and the secondary endpoints were overall survival (OS) and incidence of adverse events (AEs). Clinical outcome was compared between patients with and without cancer cachexia. A total of 53 patients were analyzed. Among all patients, median TTF and OS were significantly shorter in patients with cancer cachexia than in those without [TTF: 5.8 vs. 10 months; hazard ratio (HR): 2.13 ; 95\% confidence interval (CI): 1.07-4.24; $\mathrm{P}=0.016$; OS: 12.1 months vs. not reached; HR: 5.85 ; $95 \% \mathrm{CI}$ : $2.0-17.1 ; \mathrm{P}=0.001]$. In addition, TTF in the pembrolizumab monotherapy group was significantly shorter in patients with cancer cachexia than in those without, but no significant difference was detected in patients receiving pembrolizumab combination therapy. The incidence of AEs did not significantly differ between patients with and without cancer cachexia, except with regard to hypothyroidism. In conclusion, although cancer cachexia is prognostic of a poor outcome in patients with advanced NSCLC who receive first-line pembrolizumab,
\end{abstract}

Correspondence to: Dr Hirotoshi Iihara, Department of Pharmacy, Gifu University Hospital, 1-1 Yanagido, Gifu 501-1194, Japan E-mail: dai0920@gifu-u.ac.jp

Key words: pembrolizumab, cancer cachexia, advanced non-small cell lung cancer, overall survival, time to treatment failure cancer cachexia might not affect therapeutic efficacy in combination therapy with pembrolizumab and cytotoxic anticancer agents.

\section{Introduction}

Lung cancer is the leading cause of cancer-related mortality worldwide and the second-leading cause of new cases of cancer. Non-small cell lung cancer (NSCLC) is the most common type (1). In drug therapy for lung cancer, patients with stage IV NSCLC are treated with molecular-targeted drugs (2-9), immune checkpoint inhibitors (ICIs) (10-12) and cytotoxic anticancer drugs (13). In particular, in patients classified as driver gene mutation/rearrangement-positive, molecular-targeted drugs (kinase inhibitors) against epidermal growth factor receptor (EGFR) (2-4), anaplastic lymphoma kinase (ALK) (5), c-ROS oncogene 1 (ROS1) (6), v-raf murine sarcoma viral oncogene homologue B (BRAF) (7), and proto-oncogene cMET $(8,9)$ have shown high therapeutic efficacy. In contrast, high efficacy with pembrolizumab monotherapy or platinum combination chemotherapy plus ICI, such as programmed cell death 1 (PD-1)/programmed cell death 1-ligand 1 (PD-L1) inhibitor, has been seen in patients with driver gene mutation/rearrangement negative disease (10-12).

The introduction of pembrolizumab as an ICI, has changed the outcome of treatment drastically, extending progression-free survival (PFS) and overall survival (OS) compared to the conventional platinum-based therapy (10). The addition of pembrolizumab to conventional platinum-based therapy has also been shown to be more effective than platinum-based therapy alone $(11,12)$. While pembrolizumab monotherapy significantly extended PFS and OS only in patients with PD-L1 expression on at least $50 \%$ of tumor cells, pembrolizumab combined therapy showed efficacy even when PD-L1 expression was below $50 \%(11,12)$. Now, the main treatment of advanced NSCLC without a targetable mutation with PD-L1 expression of more than $50 \%$ is pembrolizumab, and pembrolizumab combined therapy when PD-L1 expression is less than 50\% (13). 
The efficacy of pembrolizumab and pembrolizumab combined therapy remains limited, however, and predictive markers of ICIs are important (14). Although tumor proportion score (TPS) is used to measure the expression of PD-L1 in tumor cells, its validity in predicting the effects of pembrolizumab and pembrolizumab combined therapy is also insufficient (15). Other biological features that predict high tumor expression include a high tumor mutational burden and the presence of tumor infiltrating CD8+ (16). Currently, however, only PD-L1 expression is used in routine practice, despite being an incomplete tool for prediction, as mentioned above, and new biomarkers to maximize the response of tumor regression and minimize immune-related adverse events (irAEs) are urgently needed.

Cancer cachexia is a feature of cancer that reflects the metabolic changes that occur with this condition (17). Cancer cachexia is defined as progressive skeletal muscle loss with or without weight loss that does not completely recover with conventional nutritional support and which leads to functional disability (18). The main symptom of cancer cachexia is involuntary weight loss. Cachexia is diagnosed when a weight loss greater than $5 \%$ occurs, or a weight loss greater than $2 \%$ occurs in individuals with a body mass index (BMI) below 20 or loss of skeletal muscle mass (sarcopenia) (18).

Roch et al reported that cancer sarcopenia, diagnosed by a decrease in the third lumbar vertebra skeletal muscle index (mSMI), is a useful determinant of disease control rate and survival in NSCLC patients receiving first- and second-line treatment with ICIs (19). They also reported that a body weight loss of $5 \%$ or more reduced disease control rate and OS. However, $87 \%$ of their patient population received second-line pembrolizumab monotherapy, with PD-L1 expression of $1 \%$ or more. It therefore remains unclear whether cancer cachexia predicts the efficacy of pembrolizumab in first-line treatment, in either mono- or combination therapy.

Here, we conducted a retrospective study to evaluate whether cancer cachexia is a determinant of treatment efficacy in patients receiving first-line pembrolizumab monotherapy and combined therapy.

\section{Patients and methods}

Patients. As a retrospective study, we collected data from medical records of NSCLC patients receiving first-line pembrolizumab treatment at our institution from April 2014 to June 2020. Eligibility was limited to patients treated with first-line treatment with pembrolizumab either alone or in combination with another agent.

Evaluation of cancer cachexia at the start of pembrolizumab therapy. Cancer cachexia is defined as progressive skeletal muscle loss with or without weight loss that does not completely recover by conventional nutritional support and leads to functional disability (18). Accordingly, we defined cancer cachexia as any of the following: i) weight loss greater than 5\%; ii) weight loss greater than $2 \%$ in an individual with a BMI below 20; and iii) loss of skeletal muscle mass (sarcopenia) and weight loss greater than $2 \%$. We compared weight with that 6 months prior to the day of therapy initiation as baseline. Sarcopenia was evaluated by tracing the outline of the psoas major muscle at the L2-L3 position, performed by the same single operator for all cases. The sum of the right and left areas was calculated and a change rate in psoas major muscle area (PMMA) of more than $10 \%$ was defined as sarcopenia. Change rate was defined as follows: Change rate of PMMA $(\%)=(1-P M M A ~ I C I$ initiation/PMMA before 6 months of ICI initiation) $\mathrm{x} 100$

These criteria are consistent with a study by Nishioka et al showing the association of sarcopenia and efficacy of ICI therapy in NSCLC (20).

Evaluation of pembrolizumab therapy efficacy. Time to treatment failure (TTF) was used as the primary endpoint of efficacy for pembrolizumab. We defined TTF as the time from the start of pembrolizumab therapy to the end of pembrolizumab therapy. Secondary endpoints were OS, tumor response and incidence rate of AEs. OS was defined from the start of pembrolizumab therapy to death by any cause.

Tumor response was assessed in four criteria in accordance with Response Evaluation Criteria in Solid Tumors guideline version 1.1 (21). Response rate was defined as complete response (CR) plus partial response (PR), and disease control rate as CR plus PR plus stable disease (SD).

Assessment of AEs. AEs were classified as pneumonitis, colitis, adrenal insufficiency, hypothyroidism, renal dysfunction, diabetes mellitus, hepatitis, severe skin toxicity and infusion-related reaction, and graded according to the Common Terminology Criteria for Adverse Events version 4.0 (22). Incidence rates of AEs were compared between patients with and without cancer cachexia.

Statistical analysis. Patient characteristics were summarized as medians with 25 th and 75 th percentiles for continuous variables, and frequencies and percentages for categorical variables. Differences in patient characteristics between the two groups were compared using the $\chi^{2}$ test, Fisher's exact test or Mann-Whitney U-test. For the primary analysis, a Kaplan-Meier estimate and log-rank test were used to assess OS and TTF by development of cancer cachexia. Cox proportional hazards regression was used to evaluate the association between OS and cancer cachexia with adjustment for covariates. Categorical variables such as the incidence of AEs, tumor response and one-year survival were compared between patients with and without cancer cachexia using the $\chi^{2}$ test. All analyses were conducted using IBM SPSS version 22 (IBM Japan Ltd.) and R software version 3.5.1 (www.r-project.org), with $\mathrm{P}<0.05$ considered significant.

\section{Results}

Patient demographics. A total of 53 NSCLC patients were eligible. Among them, 55\% (29/53) were diagnosed with adenocarcinoma and $32 \%(17 / 53)$ with squamous cell carcinoma. 32 patients were treated with pembrolizumab monotherapy and 21 with pembrolizumab combination therapy. Of these 21 patients, 10 patients received carboplatin plus pemetrexed, 9 received carboplatin plus nab-paclitaxel, and 2 received cisplatin plus pemetrexed other than pembrolizumab. There were 23 and 30 patients with and without cancer 
Table I. Patient demographics and baseline characteristics in patients receiving pembrolizumab with or without cancer cachexia.

\begin{tabular}{|c|c|c|c|}
\hline Characteristic & With cachexia $(n=23)$ & Without cachexia $(n=30)$ & P-value \\
\hline Number of patients with combination of cytotoxic agents & $10(43.5 \%)$ & $11(36.7 \%)$ & $0.615^{\mathrm{a}}$ \\
\hline Sex, male/female & $18 / 5$ & $24 / 6$ & $1.000^{\mathrm{a}}$ \\
\hline Age, years & $71.0(67.5-76.5)$ & $71.0(67.2-76.7)$ & $0.914^{\mathrm{b}}$ \\
\hline Height, cm & $164.9(157.4-169.6)$ & $162.2(158.9-164.9)$ & $0.290^{\mathrm{b}}$ \\
\hline Body weight, kg & $49.4(45.4-56.5)$ & $46.4(43.4-58.3)$ & $0.061^{\mathrm{b}}$ \\
\hline Body mass index & $20.9(18.5-22.6)$ & $22.2(20.8-24.5)$ & $0.002^{\mathrm{b}}$ \\
\hline Albumin, mg/dl & $3.5(3.0-3.8)$ & $4.0(3.6-4.3)$ & $0.007^{\mathrm{b}}$ \\
\hline Aspartate aminotransferase, IU/1 & $24.0(17.0-33.5)$ & $20.0(16.3-24.8)$ & $0.254^{\mathrm{b}}$ \\
\hline Alanine aminotransferase, IU/l & $23.0(12.0-40.5)$ & $16.0(12.0-26.0)$ & $0.146^{\mathrm{b}}$ \\
\hline Serum creatinine, $\mathrm{mg} / \mathrm{dl}$ & $0.64(0.61-0.73)$ & $0.79(0.60-0.90)$ & $0.068^{\mathrm{b}}$ \\
\hline Total bilirubin, mg/dl & $0.5(0.5-0.65)$ & $0.6(0.5-0.7)$ & $0.299^{\mathrm{b}}$ \\
\hline C-reactive protein, $\mathrm{mg} / \mathrm{dl}$ & $3.1(1.4-7.9)$ & $0.43(0.11-3.98)$ & $0.004^{\mathrm{b}}$ \\
\hline Neutrophils, /l & $7,840(5,342.5-9,185)$ & $4,630(3,800-5,597.5)$ & $<0.001^{\mathrm{b}}$ \\
\hline Lymphocytes, /1 & $1,210(883.5-1,355.5)$ & $1,393.5(1,115.2-1,821.2)$ & $0.032^{\mathrm{b}}$ \\
\hline White blood cells, / & $9,880(7,340-11,445)$ & $7,315(5,970-8,320)$ & $0.006^{\mathrm{b}}$ \\
\hline Hemoglobin, g/dl & $11.6(10.6-13.2)$ & $13.0(11.93-14.05)$ & $0.042^{\mathrm{b}}$ \\
\hline Platelets, $10^{4} / 1$ & $32.9(24.3-39.4)$ & $24.6(19.9-28.8)$ & $0.023^{\mathrm{b}}$ \\
\hline Modified Glasgow prognostic score, $0 / 1 / 2$ & $4 / 9 / 10$ & $19 / 6 / 5$ & $0.003^{\mathrm{a}}$ \\
\hline Neutrophil-lymphocyte ratio & $6.10(5.01-8.23)$ & $3.35(2.52-4.58)$ & $<0.001^{\mathrm{b}}$ \\
\hline Carcinoembryonic antigen, $\mathrm{U} / \mathrm{ml}$ & $4.2(2.1-19.9)$ & $5.35(1.6-36.8)$ & $0.799^{\mathrm{b}}$ \\
\hline Carbohydrate antigen 19-9, U/ml & $6.0(3.9-16.0)$ & $2.6(0.8-9.85)$ & $0.095^{\mathrm{b}}$ \\
\hline Squamous cell carcinoma antigen, $\mathrm{ng} / \mathrm{ml}$ & $2.2(1.27-14.6)$ & $1.4(1.1-2.75)$ & $0.274^{\mathrm{b}}$ \\
\hline Number of metastatic organs/sites, $0 / 1 / \geq 2$ & $8 / 9 / 6$ & $11 / 14 / 5$ & $0.371^{\mathrm{a}}$ \\
\hline Squamous cell carcinoma/Adenocarcinoma/Others & $8 / 10 / 5$ & $9 / 19 / 2$ & $0.942^{\mathrm{a}}$ \\
\hline
\end{tabular}

Data indicate medians with 25 th and 75 th percentiles or number. ${ }^{a} \chi^{2}$ test, ${ }^{b}$ Mann-Whitney U-test.

cachexia, respectively (Table I), giving an overall incidence rate of cancer cachexia at the start of pembrolizumab of $43 \%$ (23/53). As shown Table I, BMI, albumin, lymphocytes and hemoglobin were significantly lower in patients with cachexia than in those without cachexia. On the other hand, C-reactive protein (CRP), neutrophils, white blood cells, platelets and neutrophil-lymphocyte ratio (NLR) were significantly higher in patients with cachexia than in those without. On evaluation for newly arising cancer cachexia, 13 patients had a weight loss of more than $5 \%$ and 10 with a BMI below 20 had a weight loss of more than $2 \%$, meaning 23 patients met the criteria for cancer cachexia.

Efficacy of treatment. The relative dose intensity (RDI) of pembrolizumab in patients with and without cancer cachexia was 0.98 and 0.93 , respectively. Median follow up was 13.6 months (interquartile range: 2.2-6.6). For all patients who received pembrolizumab, median TTF and median OS were 6.6 months [95\% confidence interval (CI): 4.7-8.5] and 22.7 months (95\% CI: 18-27).

Median TTF and OS were significantly shorter in patients with cancer cachexia than in those without [TTF: 5.8 vs. 10 months; hazard ratio (HR): 2.13 ; 95\% CI: 1.07-4.24; $\mathrm{P}=0.016$; OS: 12.1 months vs. not reached months; HR: 5.85; 95\% CI: 2.0-17.1; $\mathrm{P}=0.001 ;$ Fig. 1].
In patients receiving pembrolizumab monotherapy, median TTF was shorter in patients with cancer cachexia than in those without. This result was not seen in patients receiving combination therapy including pembrolizumab (monotherapy: 4.2 vs. 19.4 months; HR: 3.56; 95\% CI: 1.43-8.90; $\mathrm{P}=0.007$; combination therapy: 6.5 vs. 7.3 months; HR: 1.35 ; $95 \% \mathrm{CI}$ : 0.417-4.39; $\mathrm{P}=0.615$; Fig. 2).

There was no significant difference between patients with and without cachexia in tumor response rate including response rate and disease control rate. One-year survival rate was lower in patients with cachexia than in those without (1-year survival: 26 vs. $60 \%$; $\mathrm{P}=0.029$ ) (Table II).

Incidence of AEs. Rates of pneumonitis, colitis, adrenal insufficiency, renal dysfunction, diabetes mellitus, hepatitis, severe skin toxicity and infusion-related reaction did not significantly differ between patients with and without cancer cachexia (Table III). In contrast, the rate of hypothyroidism was significantly lower in patients with cancer cachexia than in those without $(\mathrm{P}=0.048)$.

\section{Discussion}

In this study, we evaluated the impact of cancer cachexia in NSCLC patients receiving first-line treatment with 
A

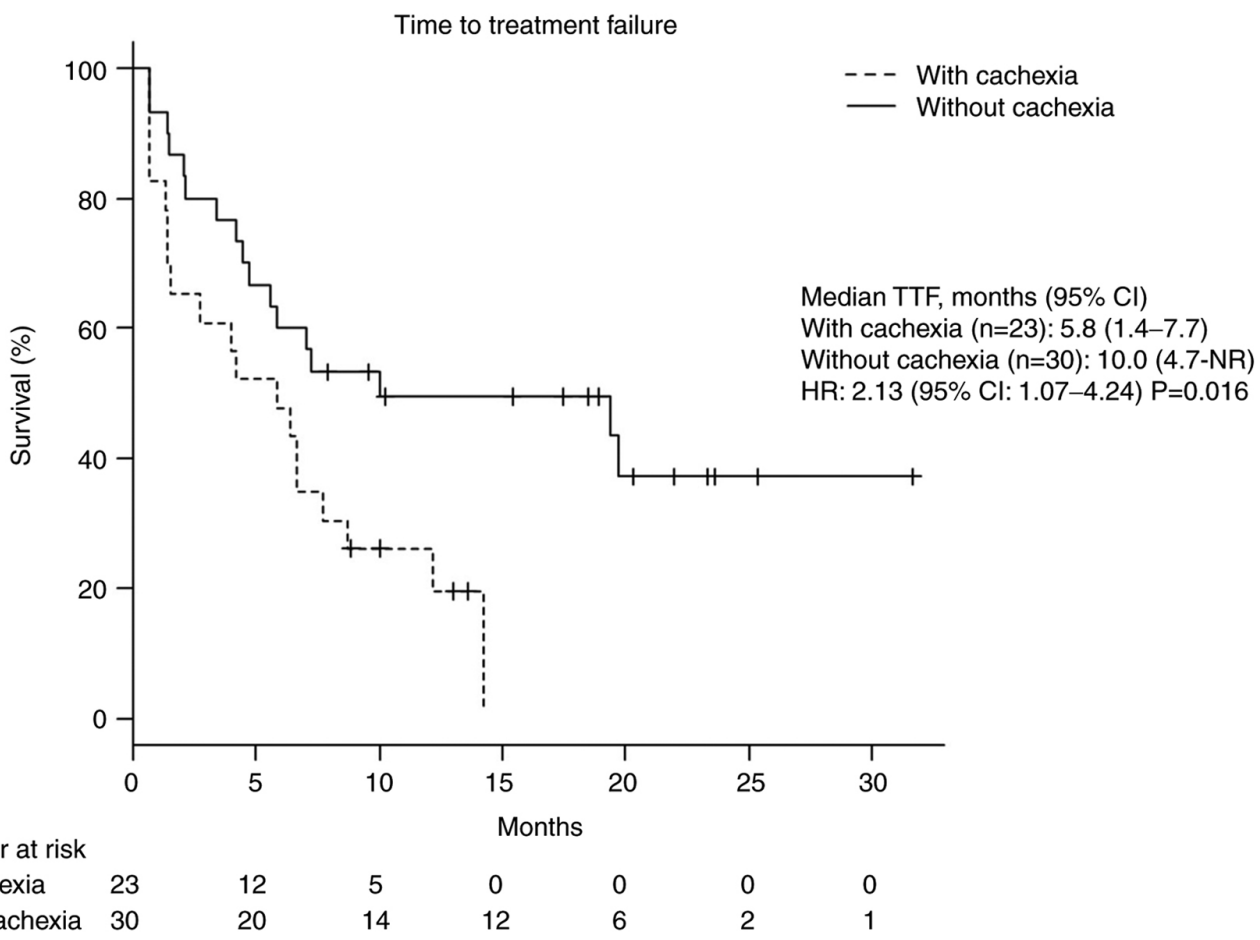

\begin{tabular}{llcccccc}
\multicolumn{7}{c}{ Number at risk } & \multicolumn{7}{c}{ Months } \\
With cachexia & 23 & 12 & 5 & 0 & 0 & 0 & 0 \\
Without cachexia & 30 & 20 & 14 & 12 & 6 & 2 & 1
\end{tabular}

B

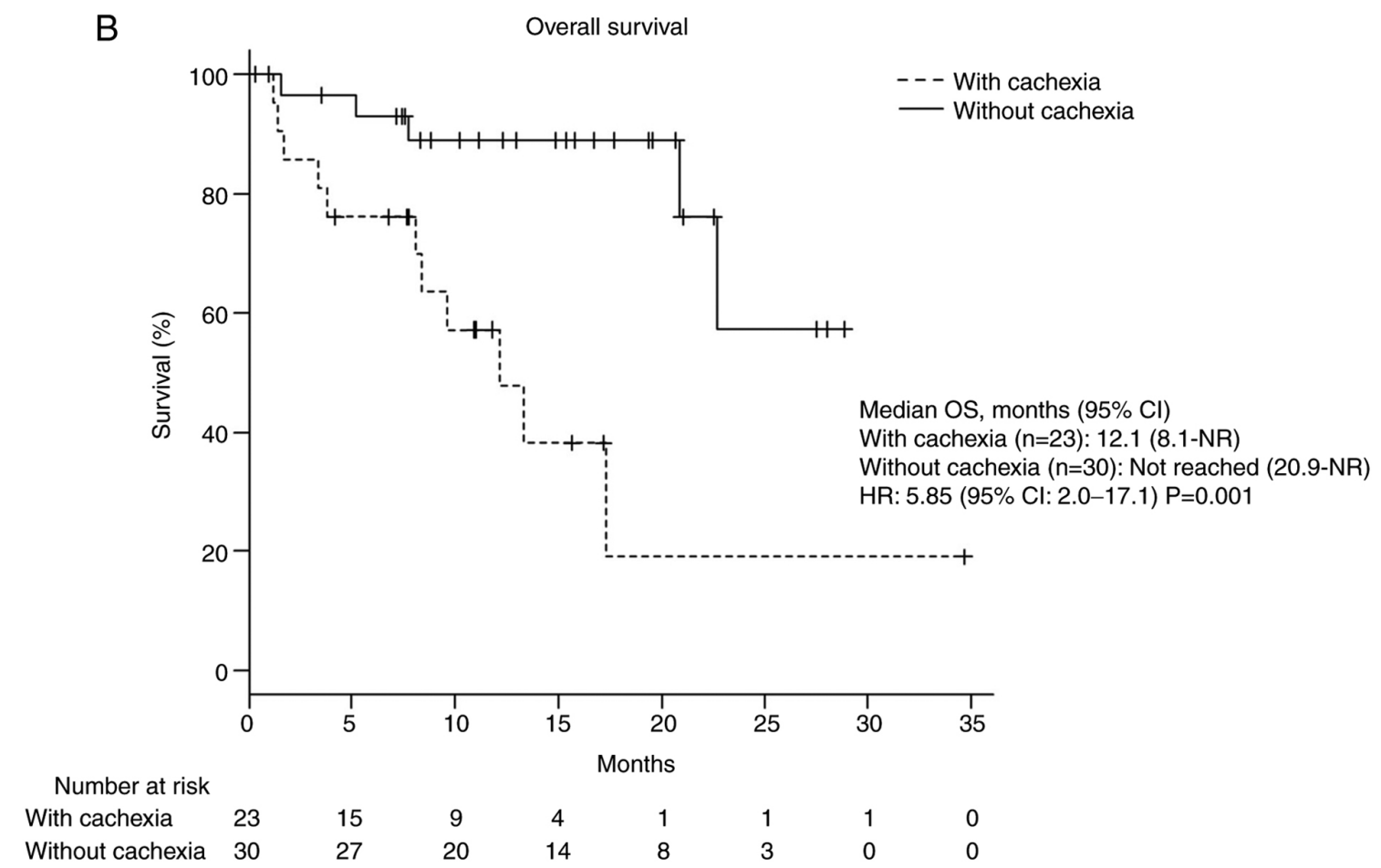

Number at risk With cachexia

Figure 1. Kaplan-Meier curves comparing (A) time to treatment failure and (B) overall survival in patients with non-small cell lung cancer receiving pembrolizumab.

pembrolizumab. Cancer cachexia was found to be predictive in these patients, and was associated with significantly shortened TTF, OS, and 1-year survival. However, no association was seen between the first-line treatment effect of pembrolizumab combined with cytotoxic anticancer agents and cancer cachexia. These findings suggest that avoidance of cachexia will not result in a weakening of the therapeutic effect of pembrolizumab monotherapy in patients with NSCLC.
In our study, TTF in patients receiving pembrolizumab was 6.6 months. This finding is inconsistent with the KEYNOTE-024 trial of Reck et al (10), who reported a PFS of 10.3 months in 305 patients with advanced NSCLC receiving pembrolizumab. It is also inconsistent with the KEYNOTE-189 trial of Gandhi et al (11), who reported a PFS of 8.8 months in 410 patients with advanced NSCLC receiving pembrolizumab in combination with pemetrexed and a platinum-based drug. 
A
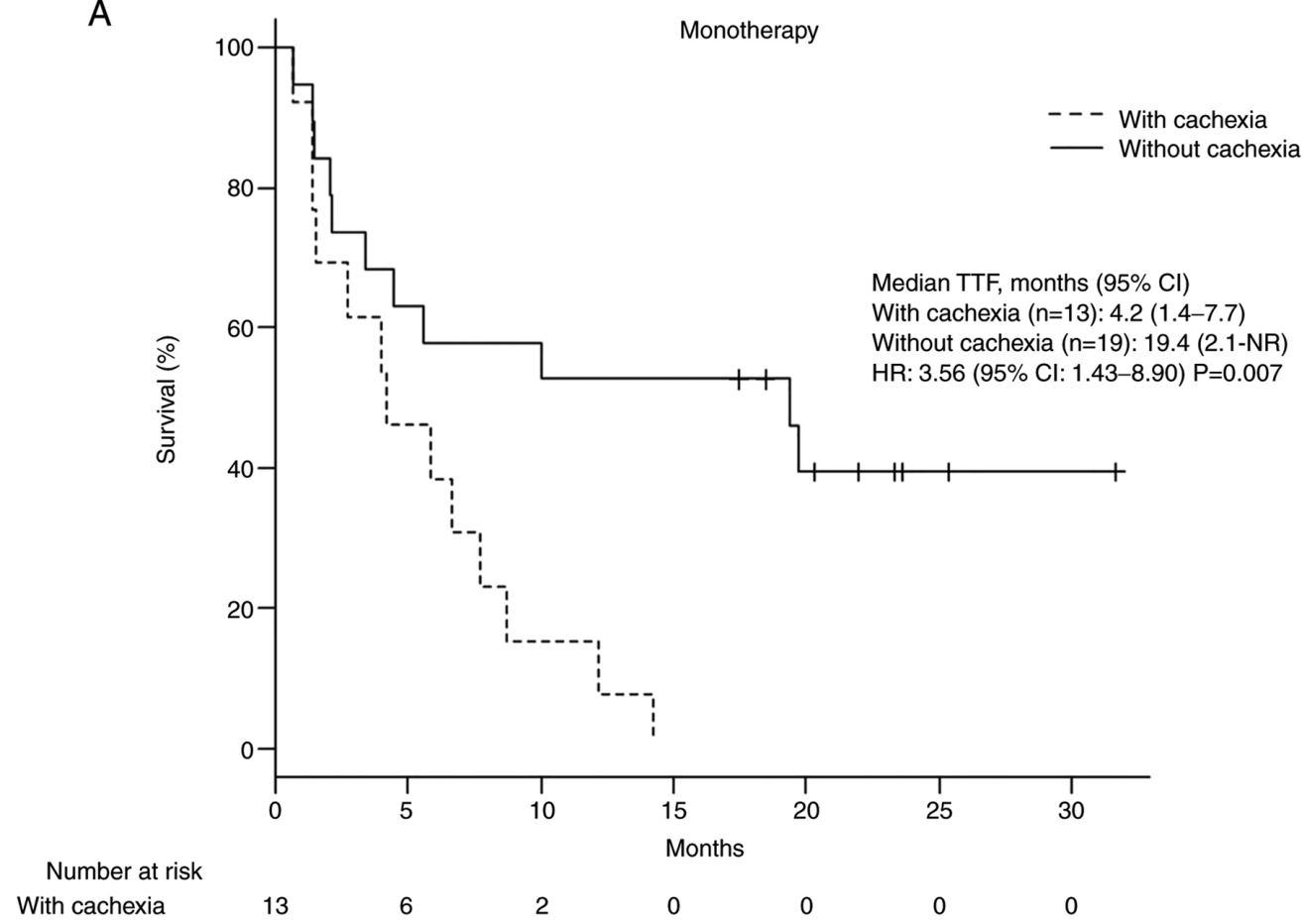

B

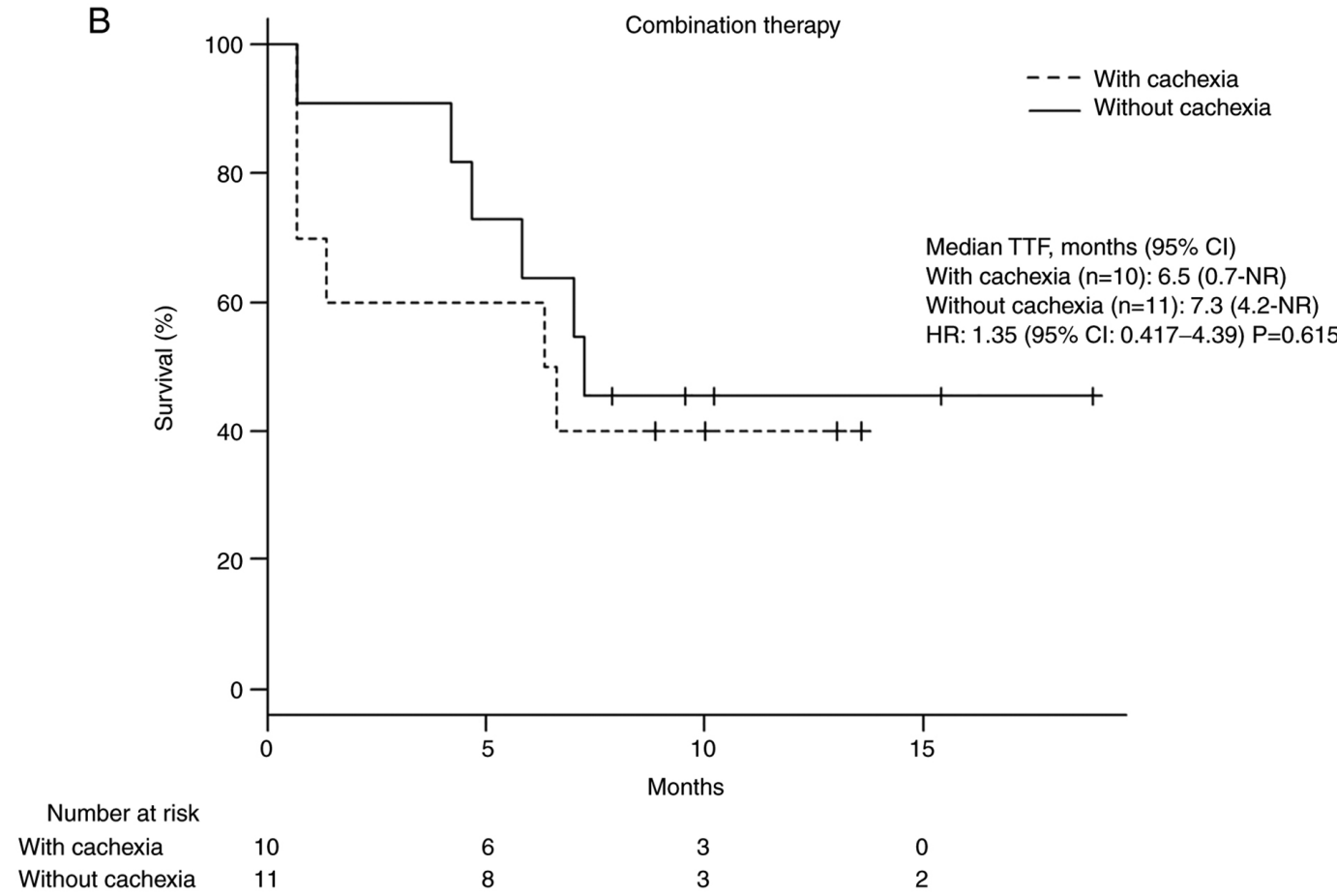

With cachexia $(n=13): 4.2(1.4-7.7)$

Without cachexia $(n=19): 19.4$ (2.1-NR)

HR: $3.56(95 \% \mathrm{Cl}: 1.43-8.90) \mathrm{P}=0.007$
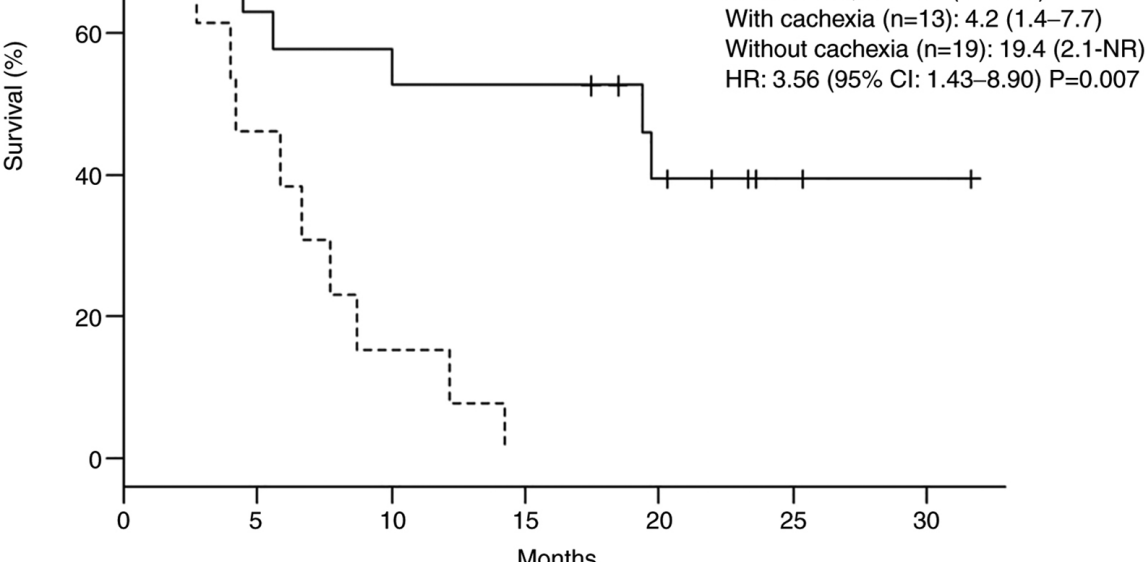

Months

Figure 2. Kaplan-Meier curves comparing time to treatment failure in patients with non-small cell lung cancer receiving pembrolizumab (A) monotherapy and (B) combination therapy.

This difference in TTF might be ascribable to recruitment: The KEYNOTE-024 and KEYNOTE-189 trials were Phase 3 clinical trials which limited recruitment to patients having adequate organ function (10). In contrast, our present study recruited all patients who received pembrolizumab in real-world clinical practice, including those in poor general condition. In addition, we considered cachexia as a factor in some patients with poor condition, whereas these are typically excluded from clinical trials. Indeed, $43.4 \%$ of our patients had cachexia. Of note, the TTF of patients who did not have cachexia (10.0 months) was generally similar to that of the pembrolizumab group (10.3 months) in the KEYNOTE-024 trial (10).

In this study, significant differences were found in BMI, albumin, CRP, neutrophil count, white blood cell count, HGB, platelets, mGPS, and NLR. Since systemic inflammation is 
Table II. Comparison of median time to treatment failure and disease control rate in patients with non-small cell lung cancer with or without cachexia.

\begin{tabular}{lccr}
\hline Effect & With cachexia $(\mathrm{n}=23)$ & Without cachexia $(\mathrm{n}=30)$ & P-value \\
\hline Tumor response rate (\%) & & & \\
Response rate (CR + PR) & $6(26.1)$ & $10(33.3)$ & $0.789^{\mathrm{a}}$ \\
Disease control rate (CR + PR + SD) & $19(82.6)$ & $26(86.7)$ & $0.715^{\mathrm{b}}$ \\
One-year survival (\%) & $6(26.1)$ & $18(60.0)$ & $0.029^{\mathrm{a}}$ \\
\hline
\end{tabular}

${ }^{\mathrm{a}} \chi^{2}$ test; ${ }^{\mathrm{b}}$ Fisher's exact test. CR, complete response; PR, partial response; SD, stable disease.

Table III. Comparison of incidence of adverse events between patients with non-small cell lung cancer with or without cachexia.

\begin{tabular}{|c|c|c|c|c|c|c|c|c|c|}
\hline \multirow[b]{2}{*}{ Adverse event } & \multicolumn{4}{|c|}{ With cachexia $(n=23)$} & \multicolumn{4}{|c|}{ Without cachexia $(n=30)$} & \multirow[b]{2}{*}{ P-value } \\
\hline & $\begin{array}{c}\text { Grade } 1 \\
(\%)\end{array}$ & $\begin{array}{c}\text { Grade } 2 \\
(\%)\end{array}$ & $\begin{array}{c}\text { Grade } 3 \\
(\%)\end{array}$ & Overall & $\begin{array}{c}\text { Grade } 1 \\
(\%)\end{array}$ & $\begin{array}{c}\text { Grade } 2 \\
(\%)\end{array}$ & $\begin{array}{c}\text { Grade } 3 \\
(\%)\end{array}$ & Overall & \\
\hline Pneumonitis & 0.0 & 0.0 & 0.0 & $0 / 23$ & 0.0 & 0.0 & 0.0 & $0 / 30$ & - \\
\hline Colitis & 4.3 & 0.0 & 0.0 & $1 / 23$ & 0.0 & 0.0 & 0.0 & $0 / 30$ & 0.434 \\
\hline Hypothyroidism & 8.7 & 0.0 & 0.0 & $2 / 23$ & 23.3 & 13.3 & 0.0 & $11 / 30$ & 0.048 \\
\hline Adrenal insufficiency & 0.0 & 0.0 & 0.0 & $0 / 23$ & 0.0 & 0.0 & 0.0 & $0 / 30$ & - \\
\hline Renal dysfunction & 4.3 & 0.0 & 0.0 & $1 / 23$ & 26.7 & 0.0 & 0.0 & $8 / 30$ & 0.061 \\
\hline Pancreatitis & 4.3 & 0.0 & 0.0 & $1 / 23$ & 3.3 & 3.3 & 0.0 & $2 / 30$ & 0.667 \\
\hline Hepatitis & 26.1 & 4.3 & 4.3 & $8 / 23$ & 40.0 & 3.3 & 0.0 & $13 / 30$ & 0.524 \\
\hline Severe skin toxicity & 21.7 & 21.7 & 0.0 & $10 / 23$ & 33.3 & 20.0 & 0.0 & $16 / 30$ & 0.642 \\
\hline Infusion-related reaction & 4.3 & 0.0 & 0.0 & $1 / 23$ & 0.0 & 0.0 & 0.0 & $0 / 30$ & 0.434 \\
\hline
\end{tabular}

Data were statistically analyzed by the $\chi^{2}$ test.

present in cachexia patients (18), CRP, neutrophil count, white blood cell count, platelet count and NLR may have been higher in cachexia patients. Low BMI, albumin, lymphocytes, and hemoglobin in patients with cancer cachexia may also be due to reduced nutritional status.

Our finding that cancer cachexia is a predictor of worse clinical outcome is consistent with previous findings by Roch et al that evolving cancer sarcopenia as determined by third lumbar vertebra skeletal muscle index is associated with a shortened OS (19). It is also consistent with the finding of Shiroyama et al that sarcopenia determined by PMI can be used to predict a poor outcome of therapy (23).

Cancer cachexia also significantly shortened TTF in patients who received pembrolizumab monotherapy. In contrast, in patients who received combination therapy which included pembrolizumab, TTF did not significantly differ between patients with and without cachexia. It is widely known that the presence of cancer cachexia shortens OS (24). This corresponds to the finding of Sanders et al that NSCLC patients with early weight loss during chemoradiotherapy had shorter OS (25). Nevertheless, Ross et al reported that NSCLC patients with weight loss receiving chemotherapy did not have significantly shorter PFS than those without weight loss (26). This raises the possibility that cytotoxic treatment failure is not associated with weight loss. Further investigation of the association between weight loss and chemotherapy failure is warranted.

Our findings indicate that cancer cachexia is strongly associated with pembrolizumab monotherapy failure. This may be the result of metabolic changes induced by cancer cachexia. The mechanism of weight loss is multifactorial, including decreased food intake, metabolic dysfunction and increased energy use (27). TNF $\alpha$ and IL- 6 have been shown to cause weight loss (26). IL-1 causes protein breakdown in skeletal muscle (27). Flint et al reported that tumor-induced IL-6 causes hypoketonemia, which in turn triggers glucocorticoids and results in immune suppression (28). These inflammatory cytokines may downregulate the efficacy of pembrolizumab. Currently, the only pharmacological treatment showing promise against cancer cachexia is anamorelin (29). Further investigation of immunotherapy downregulation may reveal the pathophysiology of cancer cachexia and lead the way to promising treatments.

The incidence of hypothyroidism was significantly higher in patients without cancer cachexia. Osorio et al reported that median OS was significantly longer in those with thyroid dysfunction than in those without in patients with NSCLC who received pembrolizumab treatment (30). Median duration 
to onset of hypothyroidism was 63 and 167 days in patients with and without cancer cachexia. It was considered that the incidence rate was lower in patients who did not have cancer cachexia due to a longer treatment period of chemotherapy including pembrolizumab.

Several limitations of our study warrant mention. It was conducted under a retrospective design at a single center. Further, the sample size was too small to allow precise consideration of confounding factors.

In conclusion, pembrolizumab monotherapy was associated with poor TTF and OS outcomes in NSCLC patients with cachexia compared to those without cachexia. Nevertheless, cachexia did not affect the clinical outcome in NSCLC patients receiving pembrolizumab plus cytotoxic anticancer agents. Improvement in cancer cachexia may improve clinical outcomes in patients with NSCLC treated with pembrolizumab monotherapy.

\section{Acknowledgements}

Not applicable.

\section{Funding}

No funding was received.

\section{Availability of data and materials}

All the datasets generated or analyzed during the present study are included in this published article.

\section{Authors' contributions}

HF and HI conceptualized this study. HF, AA and DKai acquired the clinical data. HF, AA and HI analyzed data. CH, MK, MY, JE, TI, KY, YS, TG, CS, DKaw, YK, MF, RK, YO and AS interpretated the data. YO and AS confirmed the authenticity of all the raw data. HF, AA and HI drafted the manuscript. All authors have read and approved the final manuscript.

\section{Ethics approval and consent to participate}

The study was carried out in accordance with the guidelines for human studies adopted by the Ethics Committee of Gifu University Graduate School of Medicine and the Japanese Government, and approved by the Medical Review Board of Gifu University Graduate School of Medicine (approval no. 2021-B050 Institutional Review Board). Informed consent was not obtained because this was a retrospective observational study. We posted information about the study and how patients could opt out on the website of the hospital.

\section{Patient consent for publication}

In view of the retrospective nature of the study, the need for informed consent from subjects was not mandated.

\section{Competing interests}

The authors declare that they have no competing interests.

\section{References}

1. Sung H, Ferlay J, Siegel RL, Laversanne M, Soerjomataram I, Jemal A and Bray F: Global cancer statistics 2020: GLOBOCAN estimates of incidence and mortality worldwide for 36 cancers in 185 countries. CA Cancer J Clin 71: 209-249, 2021.

2. Mitsudomi T, Morita S, Yatabe Y, Negoro S, Okamoto I, Tsurutani J, Seto T, Satouchi M, Tada H, Hirashima T, et al: Gefitinib versus cisplatin plus docetaxel in patients with non-small-cell lung cancer harbouring mutations of the epidermal growth factor receptor (WJTOG3405): An open label, randomised phase 3 trial. Lancet Oncol 11: 121-128, 2010.

3. Zhou C, Wu YL, Chen G, Feng J, Liu XQ, Wang C, Zhang S, Wang J, Zhou S, Ren S, et al: Erlotinib versus chemotherapy as first-line treatment for patients with advanced EGFR mutation-positive non-small-cell lung cancer (OPTIMAL, CTONG-0802): A multicentre, open-label, randomised, phase 3 study. Lancet Oncol 12: 735-742, 2011.

4. Sequist LV, Yang JC, Yamamoto N, O'Byrne K, Hirsh V, Mok T, Geater SL, Orlov S, Tsai CM, Boyer M, et al: Phase III study of afatinib or cisplatin plus pemetrexed in patients with metastatic lung adenocarcinoma with EGFR mutations. J Clin Oncol 31: 3327-3334, 2013.

5. Solomon BJ, Mok T, Kim DW, Wu YL, Nakagawa K, Mekhail T, Felip E, Cappuzzo F, Paolini J, Usari T, et al: First-line crizotinib versus chemotherapy in ALK-positive lung cancer. N Engl J Med 371: 2167-2177, 2014.

6. Shaw AT, Ou SH, Bang YJ, Camidge DR, Solomon BJ, Salgia R, Riely GJ, Varella-Garcia M, Shapiro GI, Costa DB, et al: Crizotinib in ROS1-rearranged non-small-cell lung cancer. N Engl J Med 371: 1963-1971, 2014.

7. Planchard D, Smit EF, Groen HJM, Mazieres J, Besse B, Helland A, Giannone V, D'Amelio AM Jr, Zhang P, Mookerjee B and Johnson BE: Dabrafenib plus trametinib in patients with previously treated BRAF (V600E)-mutant metastatic non-small cell lung cancer: An open-label, multicentre phase 2 trial. Lancet Oncol 18: 1307-1316, 2017.

8. Paik PK, Felip E, Veillon R, Sakai H, Cortot AB, Garassino MC, Mazieres J, Viteri S, Senellart H, Van Meerbeeck J, et al: Tepotinib in non-small-cell lung cancer with MET exon 14 skipping mutations. N Engl J Med 383: 931-943, 2020.

9. Wolf J, Seto T, Han JY, Reguart N, Garon EB, Groen HJM, Tan DSW, Hida T, de Jonge M, Orlov SV, et al: Capmatinib (INC280) in METAex14-mutated advanced non-small cell lung cancer (NSCLC): Efficacy data from the phase II GEOMETRY mono-1 study. J Clin Oncol 37 (Suppl 15): 9004, 2019.

10. Reck M, Rodríguez-Abreu D, Robinson AG, Hui R, Csőszi T, Fülöp A, Gottfried M, Peled N, Tafreshi A, Cuffe S, et al: Pembrolizumab versus Chemotherapy for PD-L1-Positive non-small-cell lung cancer. N Engl J Med 375: 1823-1833, 2016.

11. Gandhi L, Rodríguez-Abreu D, Gadgeel S, Esteban E, Felip E, De Angelis F, Domine M, Clingan P, Hochmair MJ, Powell SF, et al: Pembrolizumab plus chemotherapy in metastatic non-small-cell lung cancer. N Engl J Med 378: 2078-2092, 2018.

12. Paz-Ares L, Luft A, Vicente D, Tafreshi A, Gümüş M, Mazières J, Hermes B, Cay Senler F, Csôszi T, Fülöp A, et al: Pembrolizumab plus chemotherapy for squamous non-small-cell lung cancer. $\mathrm{N}$ Engl J Med 379: 2040-2051, 2018.

13. National Comprehensive Cancer Network (NCCN): Clinical Practice Guidelines in Oncology (NCCN Guidelines ${ }^{\circledR}$ ). Non-Small Cell Lung Cancer. Version 5.2021. NCCN, Plymouth Meeting, PA, 2021. https://www.nccn.org/professionals/physician_gls/pdf/nscl.pdf.

14. Nakamura Y: Biomarkers for immune checkpoint inhibitor-mediated tumor response and adverse events. Front Med (Lausanne) 6: 119, 2019.

15. Schildhaus HU: Predictive value of PD-L1 diagnostics. Pathologe 39: 498-519, 2018.

16. Lagos GG, Izar B and Rizvi NA: Beyond tumor PD-L1: Beyond tumor PD-L1: Emerging genomic biomarkers for checkpoint inhibitor immunotherapy. Am Soc Clin Oncol Educ Book 40: 1-11, 2020.

17. Chindaprasirt J: Sarcopenia in cancer patients. Asian Pac J Cancer Prev 16: 8075-8077, 2015.

18. Fearon K, Strasser F, Anker SD, Bosaeus I, Bruera E, Fainsinger RL, Jatoi A, Loprinzi C, MacDonald N, Mantovani G, et al: Definition and classification of cancer cachexia: An international consensus. Lancet Oncol 12: 489-495, 2011. 
19. Roch B, Coffy A, Jean-Baptiste S, Palaysi E, Daures JP, Pujol JL and Bommart S: Cachexia-sarcopenia as a determinant of disease control rate and survival in non-small lung cancer patients receiving immune-checkpoint inhibitors. Lung Cancer 143: 19-26, 2020.

20. Nishioka N, Uchino J, Hirai S, Katayama Y, Yoshimura A, Okura N, Tanimura K, Harita S, Imabayashi T, Chihara Y, et al: Association of sarcopenia with and efficacy of anti-PD-1/PD-L1 therapy in non-small-cell lung cancer. J Clin Med 8: 450, 2019.

21. Eisenhauer EA, Therasse P, Bogaerts J, Schwartz LH, Sargent D, Ford R, Dancey J, Arbuck S, Gwyther S, Mooney M, et al: New response evaluation criteria in solid tumours: Revised RECIST guideline (version 1.1). Eur J Cancer 45: 228-247, 2009.

22. U.S. Department of Health and Human Services, National Institutes of Health National Cancer Institute: Common Terminology Criteria for Adverse Events (CTCAE). Version 4.0 https://www.eortc.be/services/doc/ctc/. Accessed September 1, 2018.

23. Shiroyama T, Nagatomo I, Koyama S, Hirata H, Nishida S, Miyake K, Fukushima K, Shirai Y, Mitsui Y, Takata S, et al: Impact of sarcopenia in patients with advanced non-small cell lung cancer treated with PD-1 inhibitors: A preliminary retrospective study. Sci Rep 9: 2447, 2019.

24. Peixoto da Silva S, Santos JMO, Costa E Silva MP, Gil da Costa RM and Medeiros R: Cancer cachexia and its pathophysiology: Links with sarcopenia, anorexia and asthenia. J Cachexia Sarcopenia Muscle 11: 619-635, 2020.

25. Sanders KJ, Hendriks LE, Troost EG, Bootsma GP, Houben RM, Schols AM and Dingemans AM: Early weight loss during chemoradiotherapy has a detrimental impact on outcome in NSCLC. J Thorac Oncol 11: 873-879, 2016.
26. Ross PJ, Ashley S, Norton A, Priest K, Waters JS, Eisen T, Smith IE and O'Brien ME: Do patients with weight loss have a worse outcome when undergoing chemotherapy for lung cancers? Br J Cancer 90: 1905-1911, 2004.

27. Petruzzelli M and Wagner EF: Mechanisms of metabolic dysfunction in cancer-associated cachexia. Genes Dev 30: 489-501, 2016.

28. Flint TR, Janowitz T, Connell CM, Roberts EW, Denton AE, Coll AP, Jodrell DI and Fearon DT: Tumor-Induced IL-6 reprograms host metabolism to suppress anti-tumor immunity. Cell Metab 24: 672-684, 2016.

29. Katakami N, Uchino J, Yokoyama T, Naito T, Kondo M, Yamada K, Kitajima H, Yoshimori K, Sato K, Saito H, et al: Anamorelin (ONO-7643) for the treatment of patients with non-small cell lung cancer and cachexia: Results from a randomized, double-blind, placebo-controlled, multicenter study of Japanese patients (ONO-7643-04). Cancer 124: 606-616, 2018.

30. Osorio JC, Ni A, Chaft JE, Pollina R, Kasler MK, Stephens D, Rodriguez C, Cambridge L, Rizvi H, Wolchok JD, et al: Antibody-mediated thyroid dysfunction during T-cell checkpoint blockade in patients with non-small-cell lung cancer. Ann Oncol 28: 583-589, 2017.

This work is licensed under a Creative Commons Attribution-NonCommercial-NoDerivatives 4.0 International (CC BY-NC-ND 4.0) License. 